\title{
Variation of the epidermal expression of glucocorticoid receptor-beta as predictive marker of bullous pemphigoid outcome
}

Adrien BRULEFERT ${ }^{1}$, Sébastien LE JAN ${ }^{1}$, Julie PLEE ${ }^{1,2}$, Anne DURLACH ${ }^{3}$, Philippe BERNARD ${ }^{1,2}$, Frank ANTONICELLI ${ }^{1}$ and Aurélie TRUSSARDIREGNIER

$\{03$

${ }^{I}$ Laboratory of Dermatology, EA7319, IFR53, University of Reims Champagne Ardenne, Reims, France

${ }^{2}$ Department of Dermatology, Reims University Hospital, University of Reims, Reims, France

${ }^{3}$ Laboratory Pol Bouin, Maison Blanche, Reims University Hospital, Reims, France

\section{BACKGROUND}

\section{INTRODUCTION}

Systemic and local corticosteroid (CS) treatment are considered as the gold standard for treatment of bullous pemphigoid (BP). While most of patients with BP are clinically controlled upon treatment with high dose of CS within 1 or 2 weeks, about $30 \%$ of patients with BP show a relapse during the first year of treatment. In many inflammatory diseases glucocorticoid insensitivity has been associated with the up-regulation of the glucocorticoid receptor- $\beta$ (GR $\beta$ ), one of the GR isoforms. GR $\alpha$ and GR $\beta$ isoforms were shown to be expressed in skin of patients with BP. We hypothesized that GR isoform expression could differ in BP patients with on-going remission and those who relapsed during the first year of treatment

\section{OBJECTIVE}

To investigate at baseline the expression of the total GR and GR $\beta$ isoform in keratinocytes at the pri-lesional site in order to provide a potential tool to predict disease outcome

\section{METHODS}

\section{METHODS}

All peri-lesional skin biopsy specimen were obtained from the department of pathology at Reim University Hospital and performed in patients with BP before the introduction of any CS treatment. The expression of GC receptors by keratinocytes was investigated in situ by immunohistochemistry using one primary antibody directed against total GR (including GR $\alpha$ and GR $\beta$ isoforms) (sc-8992; Santa Cruz Biotechnology) and one primary specifically directed against GR $\beta$ isoform (ab130227; Abcam).

Immunostaining intensity of total GR was evaluated in the keratinocytes either in the whole cell or more precisely by discriminating between the cytoplasmic and the nuclear compartments. Values were attributed with respect to a negative control without primary antibody (value $=0$ ) up to 4 for intense labelling. The quantification was performed twice by two independent evaluators in double blind analysis.

\section{STATISTICAL ANALYSES}

Descriptive statistics such as means and SDs were performed for all quantitative measures. As the number of included BP patients in each group was smaller than 30, the studied populations could not be assumed to be normal, and then nonparametric testing, specifically Mann Whitney test, was used to compare them. The results were considered significant if $P$ values were 0.05 or less.

\section{RESULTS (1)}

Total GR expression in bullous pemphigoid
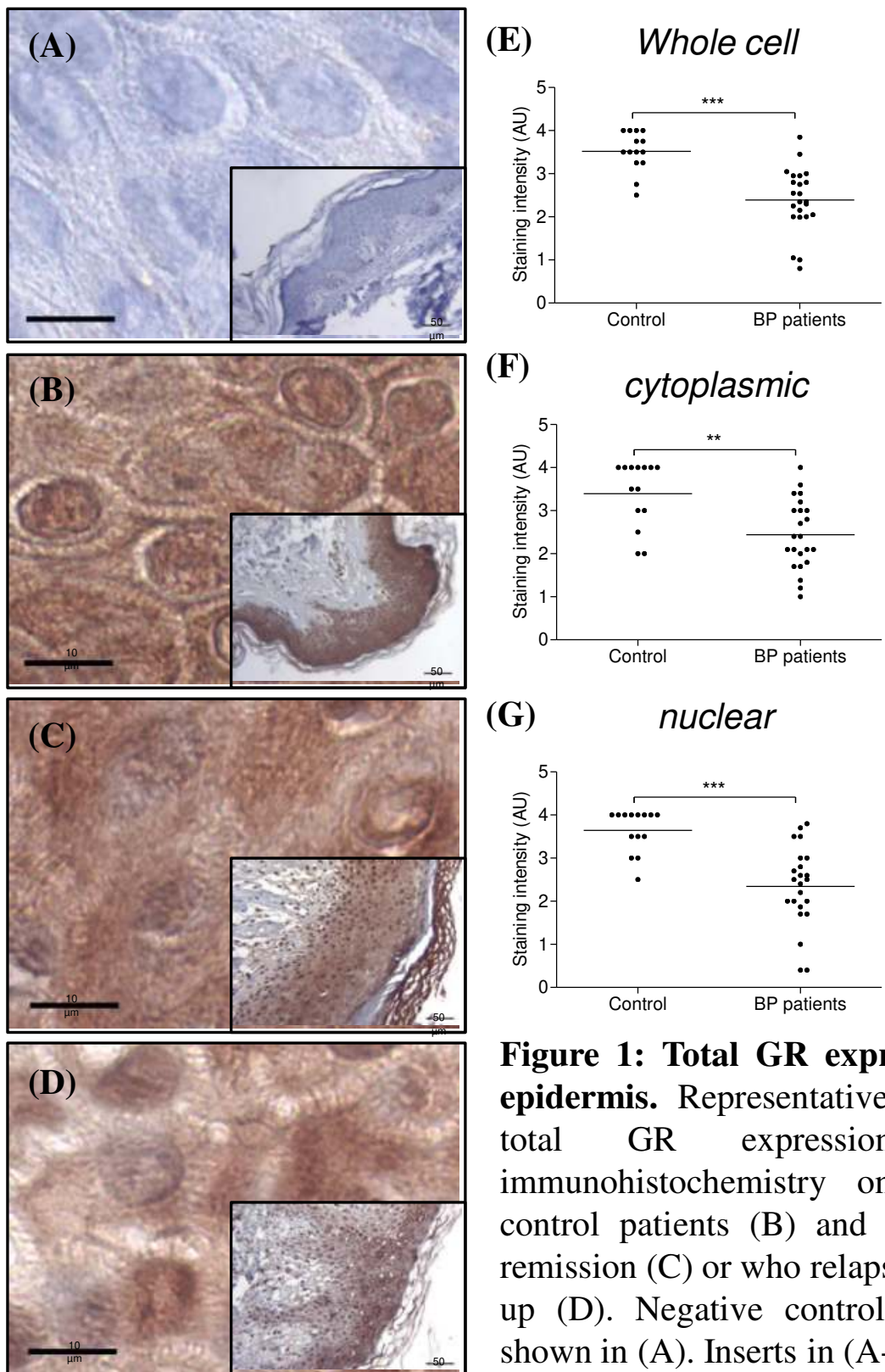

(H) Whole cell
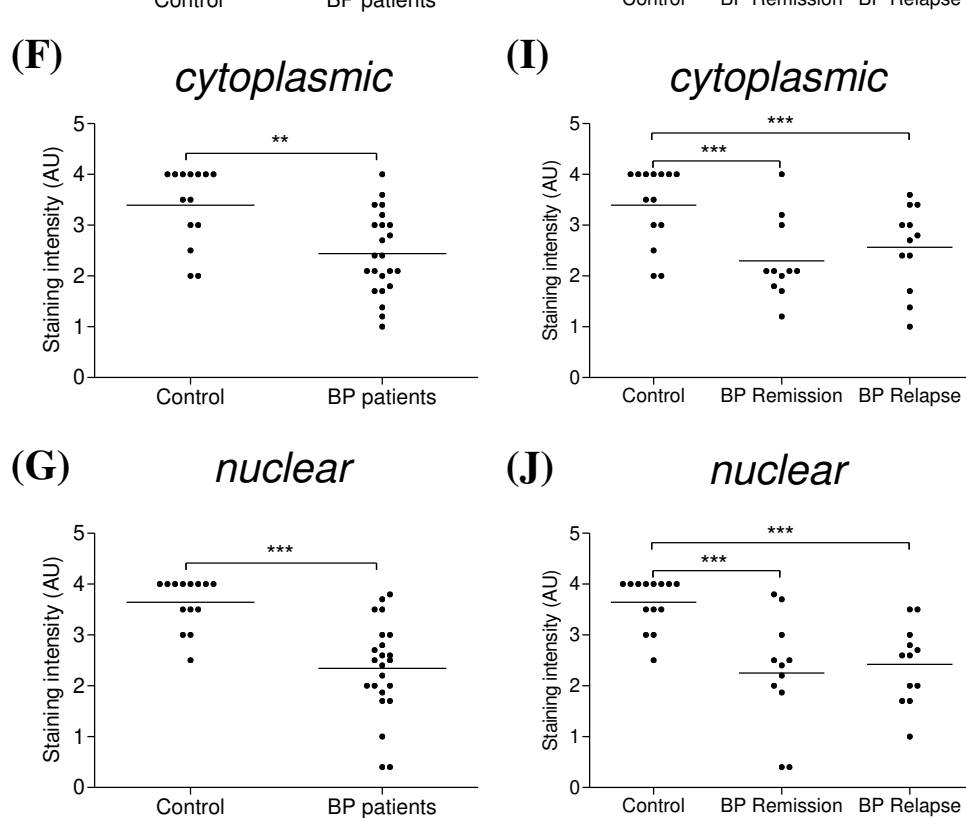

Figure 1: Total GR expression in healthy and BP skin epidermis. Representative high magnification pictures of total GR expression in keratinocytes after immunohistochemistry on skin biopsy specimen from control patients (B) and patients with BP with on-going remission (C) or who relapsed during the first year of followup (D). Negative control without primary antibody was shown in (A). Inserts in (A-D) showed low magnification picture of the area from which high magnification pictures have been taken. Staining intensity reflecting total GR expression was quantified in keratinocytes of control patients and patients with $\mathrm{BP}$ at whole cell level $(\mathrm{E}, \mathrm{H})$ or by discriminating cytoplasmic (F, I) and nuclear $(\mathrm{G}, \mathrm{J})$ compartments. Comparison was performed either between contro and total BP population (E-G) or between control patients and patients with BP according to disease outcome $(\mathrm{H}-\mathrm{J})(*, \mathrm{P}<0.05 ; * *, \mathrm{P}<0.01 ; * * *, \mathrm{P}<0.001)$

\section{PATIENTS}

26 consecutive patients with BP (according to Vaillant's well established criteria), 13 with ongoing remission and 13 with relapse within the first year of treatment, were included in this ancillary observational study conducted by the French dermatology department of Reims University Hospital. Among them, 3 patients were excluded from the study due to poor quality of the biopsies. Clinical data were recorded at baseline (Table 1). Control biopsy specimen were obtained from 14 control patients without inflammatory and autoimmune diseases. All subjects gave their informed and written consent before participating to the study in accordance with the Helsinki Declaration. Patients
without relapse Patients $P$ value Patients No Mean age + SD (range), years Mean age \pm Female/Male $75.4 \pm 20.9$

Extensive disease ${ }^{1}, \mathrm{n}(\%)$ $10(83)$

Baseline treatment

TCS

SCS

MTX

IS

Doxycyclin

Baseline comorbidities

Cardiovascular disorder

Kidney disorders

Diabetes mellitus

Neurologic diseases

Abbreviations: TCS: topical corticosteroids; SCS: systemic corticosteroids; MTX: methotrexate; IS: immunosuppressive drug

i.e. more than 10 daily blisters at diagnosi

Table 1: Baseline clinical characteristics of patients with BP

RESULTS (2)

GRß expression in bullous pemphigoid

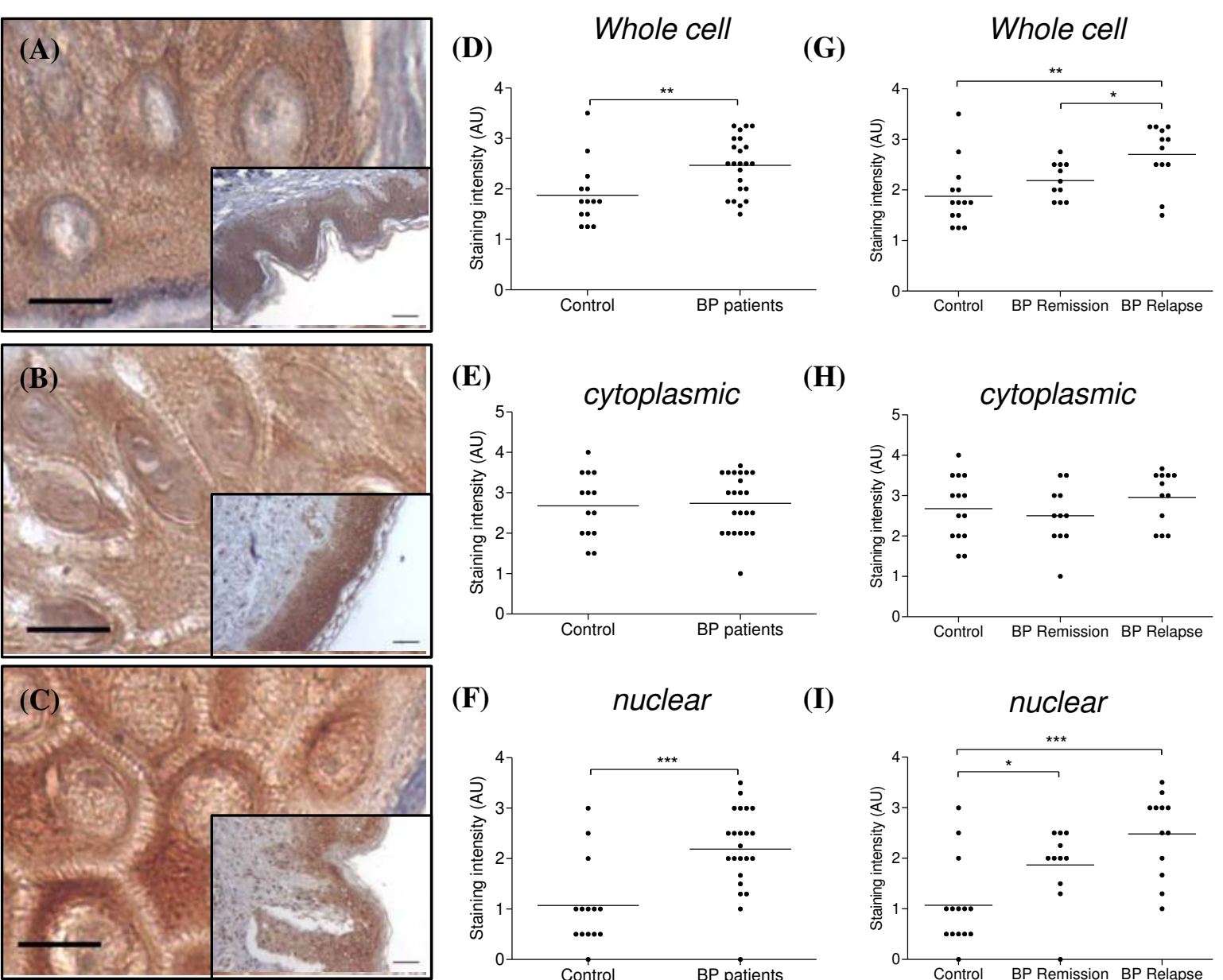

Figure 2: Increased GRß expression in BP skin epidermis. Representative high magnification photomicrographs of GR $\boldsymbol{\beta}$ expression in keratinocytes after immunohistochemistry on skin biopsy specimen from control patients (A) and patients with BP with on-going remission (B) or who relapsed during the first year of follow-up (C). Inserts in (A-C) showed low magnification picture of the area from which high magnification pictures have been taken. Bars indicate $10 \mu \mathrm{m}$ in high magnification pictures and $50 \mu \mathrm{m}$ in low magnification pictures. Staining intensity reflecting GR $\beta$ expression was quantified in keratinocytes of control patients and patients with $\mathrm{BP}$ at whole cell level $(\mathrm{D}, \mathrm{G})$ or by discriminating cytoplasmic $(\mathrm{E}, \mathrm{H})$ and nuclear $(\mathrm{F}, \mathrm{I})$ compartments. Comparison was performed either between control and total BP population (D-F) or between control patients and patients with BP according to disease outcome (G-I) $(*, \mathrm{P}<0.05$; or between control patients
$* *, \mathrm{P}<0.01 ; * * *, \mathrm{P}<0.001$ )

\section{ACKNOWLEDGEMENT}

- Champagne Ardenne Regional Council and French Department of Health for providing research grants

Plateforme IbiSA "Imagerie Cellulaire et tissulaire" for providing microscopy equipment

\section{CONCLUSION}

Overall, the present study demonstrates an increased expression of GR $\beta$ in the keratinocytes of BP patient who later relapsed compared with patients with on-going remission. These results suggest that increased expression of GR $\beta$ in the skin epithelial cells could be predictive of reduced CS treatment efficacy, and therefore of increased risk of disease relapse in the population of patients with BP. 